Revista Brasileira de Meteorologia, v.27, n.1, 13 - 22, 2012

\title{
DETERMINAÇÃO E MODELAGEM DA TAXA DE CONSUMO DE BIOMASSA QUEIMADA
}

\author{
GABRIEL PEREIRA, FRANCIELLE DA SILVA CARDOZO, FABRÍCIO BRITO SILVA, ELISABETE \\ CARIA MORAES, NELSON JESUS FERREIRA, SAULO RIBEIRO FREITAS, YOSIO EDEMIR \\ SHIMABUKURO, FÁBIO MARCELO BREUNIG, DENÍLSON RIBEIRO VIANA
}

\author{
Instituto Nacional de Pesquisas Espaciais (INPE), São José dos Campos, SP, Brasil
}

gabriel@dsr.inpe.br, cardozo@dsr.inpe.br, fabricio@dsr.inpe.br, bete@dsr.inpe.br, nelson.ferreira@dsr.inpe. br, saulofreitas@cptec.inpe.br, yosio@dsr.inpe.br, breunig@dsr.inpe.br, denilson@dsr.inpe.br

Recebido Abril de 2010 - Aceito Setembro de 2011

\begin{abstract}
RESUMO
O presente trabalho avalia o emprego da energia radiativa do fogo (ERF) para estimar as emissões de material particulado com diâmetro menor que $2,5 \mu \mathrm{m}\left(\mathrm{PM}_{2,5 \mu \mathrm{m}}\right)$, a partir da obtenção do coeficiente multiplicativo, que relaciona o consumo de biomassa com a ERF liberada. Para isto, foram utilizados dados provenientes dos produtos do MODIS (Moderate Resolution Imaging Spectroradiometer) e do produto derivado do satélite GOES (Geostationary Operational Environmental Satellite), para calcular o total de aerossóis emitidos para a atmosfera. O CCATT-BRAMS (Coupled ChemistryAerosol-Tracer Transport model coupled to Brazilian Regional Atmospheric Modelling System) foi utilizado para estimar a concentração de $\mathrm{PM}_{2,5 \mu \mathrm{m}}$ provenientes da queima de biomassa. Estes dados foram comparados com os dados de campo obtidos nos experimentos do LBA(Large Scale BiosphereAtmosphere) SMOCC (Smoke, Aerosols, Clouds, rainfall, and Climate) e RaCCI (Radiation, Cloud, and Climate Interactions). A estimativa da emissão de $\mathrm{PM}_{2,5 \mu \mathrm{m}}\left(\mu \mathrm{g} \cdot \mathrm{m}^{-3}\right)$ apresentou uma correlação com os dados do SMOCC/RaCCI superiores a 92\%. Ainda, constatou-se que o consumo máximo diário pode exceder $5 \mathrm{Tg}$ (milhões de toneladas), com uma média diária de $2,1 \mathrm{Tg}$. O método resultante das análises de laboratório permitiu estimar a biomassa consumida em $0,28 \pm 0,01 \operatorname{Pg}\left(10^{15} \mathrm{~g}\right)$ para a América do Sul, entre Julho e Novembro de 2002.
\end{abstract}

Palavras chaves: Coeficiente do consumo de biomassa, queimadas, energia radiativa do fogo.

\begin{abstract}
DETERMINATION AND MODELING THE CONSUMPTION RATE OF BURNED BIOMASS

The present study evaluates the use of fire radiative energy (FRE) to estimate the emissions of particulate matter with diameter less than $2.5 \mu \mathrm{m}\left(\mathrm{PM}_{2,5 \mu \mathrm{m}}\right)$ from the multiplicative coefficient relating the biomass consumption with the released FRE. The MODIS (Moderate Resolution Imaging Spectroradiometer) and GOES (Geostationary Operational Environmental Satellite) products were utilized to calculate the total amount of aerosol emitted to the atmosphere. The CCATT-BRAMS (Coupled ChemistryAerosol-Tracer Transport model coupled to Brazilian Regional Atmospheric Modelling System) model was used to estimate the $\mathrm{PM}_{2,5 \mu \mathrm{m}}$ concentration generated by biomass burning. The model results were compared with data obtained from the LBA (Large Scale Biosphere-Atmosphere), SMOCC (Smoke, Aerosols, Clouds, rainfall, and Climate) and RaCCI (Radiation, Cloud, and Climate Interactions) field experiments. The estimated $\mathrm{PM}_{2,5 \mu \mathrm{m}}$ emission has a correlation with the SMOCC/RaCCI data greater than $92 \%$. It was also verified that the maximum daily consumption can exceed $5 \mathrm{Tg}(5,000,000$ ton.), with a daily average of $2.1 \mathrm{Tg}$. The resulting method from laboratory analysis estimated a total of 0.28 $\pm 0.01 \mathrm{Pg}\left(10^{15} \mathrm{~g}\right)$ biomass consumed from July to November 2002 in South America.
\end{abstract}

Keywords: Biomass consumption coefficient, biomass burning, fire radiative energy. 


\section{INTRODUÇÃO}

A preocupação com a degradação ambiental é um tema muito abordado em todas as instâncias da pesquisa científica. Os processos econômicos vigentes na sociedade moderna e a intensificação da exploração dos recursos naturais, associadas a técnicas inapropriadas, produzem deteriorações irreparáveis ao meio ambiente. As alterações dos elementos naturais por práticas exploratórias relacionadas, em grande parte, com o uso e manejo da cobertura vegetal causam modificações nas interações físico-químicas e biológicas dos componentes da superfície terrestre. Estima-se que a cada ano as queimadas liberem para a atmosfera aproximadamente 100 milhões de toneladas de aerossóis (Hao e Liu, 1994).

Frequentes em todas as regiões do mundo, as queimadas consomem grande quantidade de biomassa e liberam enorme quantidade de gases traços e aerossóis para a atmosfera (Andreae e Merlet, 2001). A queima de biomassa, além de ser considerada uma das maiores fontes destes gases e de aerossóis troposféricos, modifica as características da superfície terrestre e, consequentemente, afeta o clima (Moraes et al., 2004). Dentre os principais fatores modificadores do clima destacam-se os impactos da energia liberada pelo fogo, os gases traços e aerossóis emitidos para a atmosfera, a alteração do albedo da superfície (Ichoku e Kaufman, 2005), as modificações no balanço radiativo da atmosfera e nos ciclos hidrológicos causados principalmente pelos aerossóis (Andreae et al., 2004), além de provocar problemas de saúde nas populações situadas próximas as áreas de incidência (Silva e Silva, 2006).

O impacto da queima de biomassa, no clima mundial, associada às alterações do ciclo biogeoquímico, da química atmosférica e da biodiversidade tornou-se uma grande preocupação mundial. Estudos indicam que a ocorrência de grandes incêndios tenderá a aumentar significativamente nas próximas décadas (IPCC, 2007). Além disso, alguns gases emitidos na queimada (monóxido de carbono, hidrocarbonetos não-metanos, ácido nítrico, entre outros) são quimicamente ativos e interagem com as concentrações de hidroxilas $(\mathrm{OH})$ presentes na atmosfera, alterando a eficiência de oxidação e modificando a quantidade de ozônio troposférico, que é um dos gases do efeito estufa (Levine, 1994; Kaufman et al., 1992; Galanter et al., 2000).

As informações obtidas, a partir do sensoriamento remoto ambiental, foram, por muito tempo, utilizadas apenas para detectar a distribuição espacial dos focos de queimadas e não eram utilizadas para calcular diretamente a emissão destes focos (Wooster et al., 2003). Todavia, Kaufman et al. (1996) introduziram o conceito de energia radiativa do fogo (ERF), que permite gerar dados capazes de serem relacionados diretamente com a intensidade do fogo e com o total de vegetação consumida por unidade de tempo. A ERF pode ser definida como a parte da energia química liberada na queima de biomassa emitida como radiação em consequência do processo de combustão (Wooster et al., 2003).

Diversos estudos foram realizados para se estimar a intensidade das emissões de gases provenientes da queima de biomassa (Setzer et al., 1988; Setzer e Pereira, 1988; Andreae, 1991; Kaufman et al., 1992; Kaufman et al., 1998a; Araújo et al., 1999; Badarinath et al., 2004; Andreae et al., 2004; Ichoku e Kaufman, 2005; Werf et al., 2006). Os métodos tradicionais utilizados para estimar as emissões de gases traços e aerossóis, baseiam-se na relação entre as estimativas da área queimada $\mathrm{e}$ da densidade de biomassa, multiplicada pelos fatores de emissão de cada espécie, como exemplificado na Equação 1.

$$
M^{[e]}=\alpha_{v e g} \cdot \beta_{v e g} \cdot E F_{v e g}^{[e]} \cdot a_{f o g o}
$$

onde $M[e]$ representa a emissão da espécie; $\alpha_{\mathrm{veg}}$ e $\beta_{\mathrm{veg}}$ caracterizam a fração de biomassa acima do solo e a eficiência de queima da vegetação; $E F_{\text {veg }}^{[e]}$ é o fator de emissão para cada espécie; e $a_{\text {fogo }}$ a área queimada. Embora os fatores de emissão sejam conhecidos com uma boa acurácia, atualmente existem dificuldades para determinar quantitativamente os demais fatores envolvidos (Ichoku e Kaufman, 2005).

Desta forma, essas estimativas das emissões originadas pelas queimadas devem incorporar fatores como o tamanho das áreas queimadas e as áreas mínimas detectadas pelos sensores remotos, assim como, gerar dados em tempo quasereal para serem utilizados como dados de entradas em modelos ambientais, como, por exemplo, o CCATT-BRAMS (Coupled Chemistry-Aerosol-Tracer Transport model coupled to Brazilian Regional Atmospheric Modelling System), que fornece prognósticos de poluição do ar.

O presente trabalho tem como objetivo determinar, a partir da relação experimental entre a ERF e o processo de combustão, o coeficiente de biomassa consumida a partir de análises espectroradiométricas obtidas em laboratório, e aplicar este coeficiente nas estimativas de energia radiativa do fogo derivadas do sensor MODIS e do GOES para calcular as emissões de material particulado com diâmetro menor que 2,5 $\mu \mathrm{m}\left(\mathrm{PM}_{2,5 \mu \mathrm{m}}\right)$ para o período de queimadas de 2002 na América do Sul, e modelar estas emissões no CCATT-BRAMS.

\section{MATERIAIS E MÉTODOS}

\subsection{Produtos MOD14/MYD14 - MODIS e WFABBA/GOES}

Os produtos de fogo MOD14 e MYD14 (MODIS Thermal Anomalies / Fire 5-MIN L2 SWATH 1KM V005 nível 2) são disponibilizados pelo EOS Data Gateway (http:// 
edcimswww.cr.usgs.gov/pub/imswelcome). Estes produtos incluem informações de fogos ativos e outras anomalias termais, e cobrem uma área de aproximadamente 2330 por $2030 \mathrm{~km}$ no sentido across e along-track, respectivamente, com resolução espacial nominal de $1 \mathrm{~km}$ (Giglio, 2005). Os produtos MOD14 e MYD14 são disponibilizados em formado HDF e suas informações separadas pelos SDS (scientific data set).

O WFABBA (Wildfire Automated Biomass Burning Algorithm) é um produto para detecção de anomalias termais/ fogo baseado no satélite GOES, disponibilizado com uma alta freqüência de observações, numa resolução espacial nominal de 4 x 4 km no NADIR. O algoritmo do WFABBA, assim como no MODIS, utiliza duas bandas para a detecção do pixel com

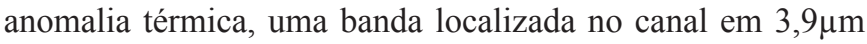
e outra localizada em 10,7 $\mu \mathrm{m}$ (Prins et al., 1998). Como os produtos de queimada WFABBA ainda não possuem um algoritmo para se estimar a ERF, estuda-se a possibilidade de adotar a Equação 2 para o cálculo desta grandeza física.

$$
E R F=A \cdot \sigma \cdot T_{f}^{4}
$$

onde A representa a fração de área de fogo no pixel, $\sigma$ é a constante de Stefan-Boltzmann $\left(5,67 \times 10^{-8} \mathrm{~J} \cdot \mathrm{s}^{-1} \cdot \mathrm{m}^{-2} \cdot \mathrm{K}^{-4}\right)$ e $T_{f}^{4}$ é a temperatura da fração do sub-pixel com fogo elevada a quarta potência. Em ambos os casos, a área e a temperatura do fogo são derivadas pelo método de Dozier (1981).

\subsection{FieldSpec Pro FR}

O FieldSpec Pro FR, fabricado pela empresa norteamericana Analytical Spectral Devices, foi utilizado no experimento de queimada realizado no Instituto Nacional de Pesquisas Espaciais (INPE), próximo ao Laboratório de Radiometria (LARAD). O intervalo espectral medido pelo FieldSpec Pro FR é de 350 a $2500 \mathrm{~nm}$ com um campo de visada de $25^{\circ}$, sua resolução espectral é de 3 a 10 nm e o tempo para aquisição de dados é de aproximadamente 1/10 de segundo por espectro.

\subsection{A energia radiativa do fogo (ERF)}

Kaufman et al. (1996) introduziram o conceito de Energia Radiativa do Fogo (correspondente a integração da potência radiativa do fogo), que permite calcular a parte da energia química liberada na queima de biomassa e emitida como radiação durante o processo de combustão. Além disso, a ERF permite gerar dados capazes de serem relacionados com a intensidade do fogo e com o total de vegetação consumida por unidade de tempo (Wooster et al., 2003; Kaufman et al., 1998a).

Os primeiros estudos sobre a ERF foram realizados utilizando o MAS (MODIS Airborne Simulator) nos experimentos SCAR-C e SCAR-B (Smoke, Cloud and Radiation, Californial
Brazil) (Kaufman et al., 1996, 1998a, 1998b; Chu et al., 1998). Os resultados já demonstravam que as estimativas das emissões através da ERF eram mais confiáveis do que as estimativas por focos de queimada.

A ERF pode ser obtida de várias maneiras: Kaufman et al. (1996) usaram uma relação semi-empírica entre a ERF e a radiância espectral do infravermelho, referente à banda 21 do MODIS (centrada em $4 \mu \mathrm{m}$ ), associando, desta forma, a ERF com a temperatura de brilho do pixel com anomalia térmica $\left(\mathrm{T}_{\mathrm{f} 4 \mu \mathrm{m}}\right)$ e com a temperatura do background $\left(\mathrm{T}_{\mathrm{b} 4 \mu \mathrm{m}}\right)$ da respectiva banda. A Equação 3 representa a ERF obtida através do sensor MODIS.

$$
\mathrm{ERF}=\left[4,3 \times 10^{-19} \cdot\left(\mathrm{T}_{\mathrm{f} 4 \mu \mathrm{m}}^{8}-\mathrm{T}_{\mathrm{b} 4 \mu \mathrm{m}}^{8}\right)\right] * \mathrm{~A}_{\mathrm{sampl}}
$$

onde $\mathrm{A}_{\text {sampl }}$ representa o tamanho da área do pixel $\left(\mathrm{km}^{2}\right)$. Pode-se ainda obter a ERF a partir de outras formulações, como exemplificado nas Equações 4 e 5 (Wooster et al., 2003):

$$
\begin{aligned}
& E R F=\mathrm{A}_{\mathrm{sampl}} \cdot \sigma \cdot \varepsilon \cdot \Sigma \mathrm{A}_{\mathrm{n}} \cdot \mathrm{T}_{\mathrm{n}}{ }^{4} \\
& E R F_{\mathrm{IVM}}=\left(\mathrm{A}_{\mathrm{sampl}} \cdot \sigma \cdot \varepsilon / \mathrm{a} \cdot \varepsilon_{\mathrm{ivm}}\right)^{*} \mathrm{~L}_{\mathrm{IVM}, \mathrm{f}}
\end{aligned}
$$

na qual $\mathrm{A}_{\mathrm{n}}$ é a área parcial do enésimo (n) componente termal no interior do pixel, $\mathrm{T}_{\mathrm{n}}$ é a temperatura da enésima componente termal $(\mathrm{K}), \varepsilon$ é a emissividade da superfície estudada, $\mathrm{ERF}_{\mathrm{IVM}}$ representam a energia radiativa do fogo para o infravermelho médio, a é uma constante obtida por Wooster e Rothery (1997), que dependem do comprimento de onda e do intervalo térmico utilizado, e $\mathrm{L}_{\mathrm{IVM}, \mathrm{f}}$ a radiância espectral do infravermelho médio para o pixel com anomalia térmica.

\subsection{Descrição do experimento ERF}

O experimento para medir a ERF e a taxa de consumo do composto orgânico ocorreu no dia 29/Nov/2007 próximo ao LARAD/INPE. Realizou-se o experimento no período noturno sem a influência de luz artificial e interferência da radiação solar no sinal coletado. Na hora do experimento, as $20 \mathrm{hrs}$, a condição meteorológica era predominantemente de céu limpo com a temperatura instantânea do ar de $20,4^{\circ} \mathrm{C}$, umidade relativa de $60 \%$ e ventos de 2 a $8 \mathrm{~km} / \mathrm{h}$.

Foram expostas aos processos de combustão duas espécies vegetais diferentes: uma proveniente do Pantanal-SulMatogrossense e outra da região Sudeste. A vegetação do bioma de cerrado, correspondente a uma espécie de gramínea muito encontrada em campos limpos e áreas de pastagem, foi obtida três meses antes do experimento, tempo suficiente para que esta perdesse grande parte da umidade presente em sua estrutura e, deste modo, reproduzir os padrões encontrados no período de seca naquela região.

Para posicionar os equipamentos destinados a medir a radiância emitida e calcular a taxa de consumo, montou-se 
um andaime com aproximadamente 4 metros de altura (Figura 1a). Nesta plataforma, posicionou-se o Fieldspec Pro FR (Figura 1b) a uma distância de $110 \mathrm{~cm}$ e $265 \mathrm{~cm}$ das amostras da biomassa vegetal prontas para o processo de combustão (Figura 1c). O instantaneous field of view (IFOV) do instrumento foi preenchido com as amostras de biomassa para não haver contaminação do fundo no sinal coletado. Após estes procedimentos, a biomassa foi pesada a cada intervalo de 10 segundos em uma balança (Figura 1d), indicando desta forma, o total de biomassa consumida por unidade de tempo.

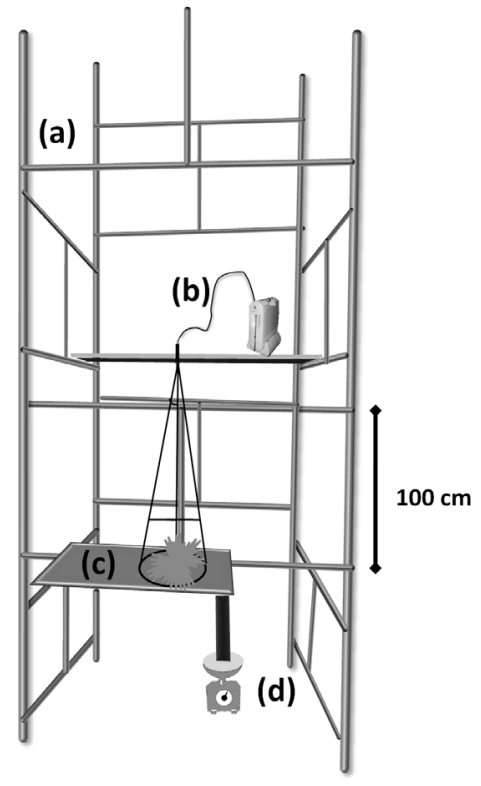

Figura 1 - Estrutura montada para o campo e posicionamento dos equipamentos. (a) Estrutura; (b) FieldSpec Pro FR; (c) Amostras de vegetação; e (d) Balança.

Pela inversão da lei de Planck foi obtida a temperatura de brilho do fogo. A Equação 6 mostra o cálculo da temperatura para um dado comprimento de onda e sua respectiva radiância.

$$
\mathrm{T}(\lambda, \mathrm{T})=\left\{\left[1,43879 \times 10^{4} / \lambda \cdot \ln \left[\left(1,17543 \times 10^{9} / \lambda \cdot \mathrm{L}(\lambda, \mathrm{T})\right)\right]\right\}\right.
$$

onde $\lambda$ representa o comprimento de onda e $L(\lambda, T)$ a radiância espectral.

\subsection{Modelo CCATT-BRAMS}

O modelo CCATT-BRAMS (Coupled ChemistryAerosol-Tracer Transport model coupled to Brazilian Regional Atmospheric Modelling System), desenvolvido para simular a circulação atmosférica em várias escalas, baseia-se no modelo numérico de previsão do tempo BRAMS, originado do modelo RAMS (Walko et al., 2000).

O CCATT, modelo de transporte Euleriano acoplado ao BRAMS, é um modelo numérico que simula e estuda os processos e transportes associados à emissão de queimadas. $\mathrm{O}$ transporte dos gases traço e aerossóis são feitos simultaneamente com a evolução do estado atmosférico, utilizando os mesmos tempos de simulação e as mesmas parametrizações dinâmicas e físicas da atmosfera. A equação de conservação de massa para o $\mathrm{PM}_{2,5 \mu \mathrm{m}}$ é calculada, sob a forma de equação de tendência, pela Equação 7 (Freitas et al., 2009):

$$
\begin{gathered}
\frac{\partial \bar{s}}{\partial t}=\left(\frac{\partial \bar{s}}{\partial t}\right)_{\mathrm{adv}}+\left(\frac{\partial \bar{s}}{\partial t}\right)_{\mathrm{PBL} t \text { turb }}+\left(\frac{\partial \bar{s}}{\partial t}\right)_{\text {Conv. Rasa }}+\left(\frac{\partial \bar{s}}{\partial t}\right)_{\text {Conv. Prof }}+ \\
W_{\mathrm{PM}_{2,5 \mu \mathrm{m}}}+R+Q
\end{gathered}
$$

onde $\frac{\partial \bar{s}}{\partial t}$ representa a tendência local, $a d v$ a advecção na escala da grade, $P B L$ turb o transporte turbulento na camada limite planetária na sub-grade, Conv. Rasa o transporte em sub-grade associado com a convecção rasa de umidade, Conv. Prof o transporte em sub-grade associado com a convecção profunda de umidade, $W_{\mathrm{PM}_{2.5 u \mathrm{~m}}}$ a remoção convectiva úmida para o material particulado, $R$ o termo associado com a remoção seca genérica e/ou transformações químicas e $Q$ a fonte de emissão associada com o processo de queimada (Freitas et al., 2009).

As simulações de emissão, para o $\mathrm{PM}_{2,5 \mu \mathrm{m}}$ feitas no CCATT-BRAMS no período de 2002 (15/julho a 15/Nov), tiveram como configurações de entrada uma grade com 50 km de resolução horizontal cobrindo toda a América do Sul. A resolução vertical foi ajustada para variar a uma taxa de 1,07 a partir de $150 \mathrm{~m}$, modelando os dados até uma altitude de 20.180 metros em 38 níveis verticais. O modelo de solos foi inicializado com 7 camadas, distribuídas nos primeiros 4 metros de profundidade (Freitas et al., 2009). O tempo total de integração no modelo foi de 124 dias, de $15 / \mathrm{Jul} / 2002$ as 00:00 UTC ao dia 16/Nov/2002 as 00:00 UTC. Para estes dias adotaram-se as condições iniciais da atmosfera e as condições de contorno provenientes do modelo ECMWF (European Center for Medium range Weather Forecasting) e do modelo do CPTEC, denominado GAMRAMST062, para as datas de $15 / \mathrm{Jul} / 2002$ a $31 / \mathrm{Ago} / 2002$; e $01 / \mathrm{Set} / 2002$ a $16 / \mathrm{Nov} / 2002$, respectivamente.

Para avaliar o desempenho das simulações realizadas no CCATT-BRAMS, os resultados foram comparados com os dados de campo coletados na região de Rondônia e Mato Grosso descritos em Freitas et al. (2009). Estes dados fazem parte dos experimentos do LBA (Large Scale Biosphere-Atmosphere), SMOCC (Smoke, Aerosols, Clouds, rainfall, and Climate) e RaCCI (Radiation, Cloud, and Climate Interactions), medidos na região amazônica no período de queimadas de 2002.

\section{RESULTADOS E DISCUSSÕES}

\subsection{Respostas espectrais e a radiância liberada}


A Figura 2 apresenta o comportamento espectral das amostras de vegetação submetidas ao processo de combustão no experimento com a ERF e a resposta espectral da placa Spectralon, com reflectância de aproximadamente $100 \%$ em todo o espectro. Em comprimentos de onda superiores a 2150 $\mathrm{nm}$ visualizam-se alguns ruídos, inerentes ao equipamento.

As amostras da vegetação de campos/pastagens, obtidas no Pantanal Sul-Mato-Grossense (em cinza claro) referentes ao Bioma Cerrado, encontravam-se em avançado estágio de senescência, com elevados valores de fator de reflectância no infravermelho próximo e médio (800 a $1500 \mathrm{~nm}$ ). Ainda, o comportamento espectral no visível (400 a $700 \mathrm{~nm}$ ) apresentou valores mais elevados na faixa espectral do amarelo e vermelho, caracterizando a ausência de pigmentação ativa.

As amostras de vegetação extraídas do INPE, uma semana antes do processo de combustão, estão representadas na cor cinza escuro. Percebe-se uma grande diferença na resposta espectral na região do visível, com picos de reflectância na faixa do espectro eletromagnético correspondente ao verde $(550 \mathrm{~nm})$ e com feições de absorção na faixa espectral correspondente ao vermelho, denotando a presença de pigmentos. Na faixa espectral do infravermelho próximo e médio $(750 \mathrm{a} 1100 \mathrm{~nm}$ ) foram encontrados elevados valores de fator de reflectância. A partir de $1350 \mathrm{~nm}$, próximos as bandas de absorção de água, as repostas espectrais das vegetações do Pantanal e do INPE foram semelhantes.

A Figura 3 apresenta o comportamento espectral da radiância emitida no experimento de queima controlada de 400 gramas de biomassa vegetal proveniente do Pantanal Sul Mato-Grossense. Embora a curva represente um caso específico de queima, as outras 15 amostras apresentam comportamento similar, diferenciado apenas na intensidade e duração dos valores obtidos.
Observa-se no gráfico (Figura 3) a evolução temporal da radiância emitida no processo de queima da biomassa vegetal. A aquisição dos dados no período noturno faz com que a radiância inicial no $\mathrm{t}=0$ apresente valores baixos, praticamente iguais a zero. A segunda medida radiométrica, geralmente 10 segundos após o início do processo de combustão, mostra que embora apenas uma pequena parcela do fogo afete a área do IFOV do sensor, esta pequena contribuição pode ser detectada. À medida que as chamas aumentam sua intensidade e cobrem uma área maior do IFOV, percebe-se uma elevação nos valores de radiância nos comprimentos de onda superiores a $2300 \mathrm{~nm}$.

As análises das radiâncias espectrais, obtidas nos 16 experimentos a cada 5 segundos, mostram que a partir do momento em que as chamas cobrem todo o IFOV do sensor, geralmente depois de 40 a 50 segundos do início da combustão, os valores de radiância espectral não sofrem alterações significativas, permanecendo desta forma, constantes. Neste período, percebe-se uma elevação nos valores de radiância na região do infravermelho médio (2300 a $2500 \mathrm{~nm})$, relacionado com a emissão da radiância de acordo com a temperatura.

A Figura 3 mostra que após a extinção de grande parte da biomassa disponível, os valores de radiância sofrem uma redução gradual com o decorrer do tempo. Entre os principais fatores desta redução está a diminuição das chamas e o início da fase de brasas, onde a temperatura e o consumo de biomassa são menores.

\subsection{Relação entre o consumo de biomassa e a ERF}

A Figura 4 mostra o resultado final dos experimentos que relacionam o total de biomassa consumida e a ERF liberada neste processo. Atualmente, encontram-se na literatura alguns coeficientes que multiplicados pela ERF permitem calcular o total de biomassa consumida pela queimada, podendo-se citar o

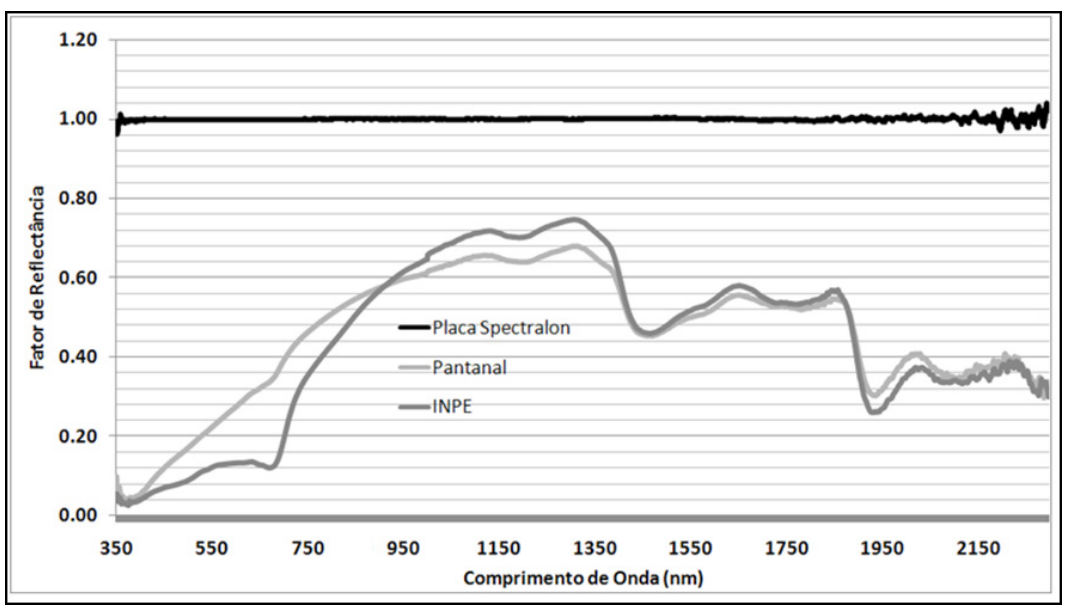

Figura 2 - Comportamento Espectral da vegetação colhida no Pantanal (cinza claro), da vegetação retirada no INPE (cinza escuro) e da placa Spectralon (preta). 


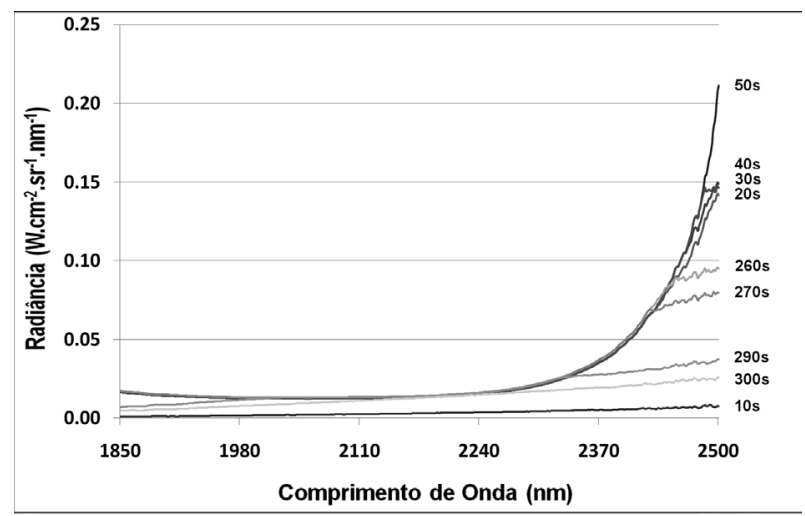

Figura 3 - Exemplo de amostras da radiância espectral de um experimento no intervalo de tempo de 10 a 300 segundos após o início do processo de combustão.

coeficiente encontrado por Wooster et al. (2005) e por Freeborn et al. (2008).

Com um coeficiente de determinação de 0,73 (significativo de acordo com o teste t de student, $\alpha=0,05$ ) o valor do coeficiente de multiplicação encontrado neste trabalho foi de $0,949\left(\mathrm{em} \mathrm{kg} . \mathrm{s}^{-1} \cdot \mathrm{MJ}^{-1}\right)$, ou seja, para cada MJ. $\mathrm{s}^{-1}$ liberado pela queimada é consumido aproximadamente $1 \mathrm{~kg}$ de biomassa vegetal. Como descrito em Wooster et al. (2005), os coeficientes encontrados em experimentos de combustão de pequena escala devem sofrer algumas alterações para serem aplicados aos dados de satélites, como, por exemplo, o MODIS e o GOES. A relação entre o consumo de biomassa e a energia radiativa liberada pode ser calculada através da Equação 8:

$$
\mathrm{BC}=0,949 * \mathrm{ERF}
$$

onde $\mathrm{BC}$ representa a quantidade de biomassa consumida em $\mathrm{kg} . \mathrm{s}^{-1}$ e ERF a energia radiativa do fogo em MJ.s $\mathrm{s}^{-1}$. Para se obter a quantidade de biomassa consumida pelas queimadas integraram-se os dados com uma persistência de 1 hora, ou seja, para cada foco detectado pelo WFABBA/GOES e MOD14 e MYD14/MODIS estimou-se o período de queima em uma hora.

\subsection{Modelagem das emissões no CCATT-BRAMS}

Com os experimentos realizados no LARAD, definiu-se um coeficiente multiplicativo semelhante aos propostos por Wooster et al. (2005) e Freeborn et al. (2008), a diferença deste coeficiente está na relação entre a quantidade de biomassa consumida e a ERF liberada. Enquanto que, o coeficiente proposto inicialmente por Wooster et al. (2005) é de 0,368 e o coeficiente encontrado por Freeborn et al. (2008) é de 0,710; encontrou-se no experimento realizado no INPE o valor de 0,949 , ou seja, aproximadamente $158 \%$ e $34 \%$ maiores que os encontrados anteriormente. Alguns fatores responsáveis por estas incertezas decorrem das observações simultâneas do foco de queimada e da fumaça liberada (erro aproximado de $\pm 11 \%$ ), a acurácia e consistência da FRP $( \pm 16 \%)$, entre outros fatores.

Para verificar a duração de cada foco do MODIS originou-se uma matriz de dados para o MODIS e para o WFABBA, representadas em grades de $4 \mathrm{~km}$ com coordenadas geográficas comum à ambos os produtos. Posteriormente, as duas grades são comparadas e se os focos detectados pelo MODIS, agora agrupados na resolução da grade, forem observados pelo WFABBA no intervalo de 3 horas (90 minutos anteriores e posteriores), um contador é iniciado e incrementado, indicando que houve a observação. Ao final, as emissões totais dos focos dos produtos MOD14/MYD14 são calculadas pela multiplicação do número de contadores pela emissão original calculada na passagem do MODIS.

A emissão de $\mathrm{PM}_{2,5 \mu \mathrm{m}}$ no período de 15/Jul/2002 a 15/ Nov/2002, estimada com o uso do coeficiente multiplicativo obtido através dos experimentos realizados no INPE é apresentada na Figura 5. Na análise dos dados, observa-se que esta metodologia, com correlação de 0,92 entre os dados modelados e os dados coletados em campo $(\alpha=0,05)$, apresentou uma subestimativa de aproximadamente $12 \%$ no valor modelado de $\mathrm{PM}_{2,5 \mu \mathrm{m}}$. No mapa do total emitido entre o período analisado, os maiores valores foram encontrados nos Estados do Mato Grosso, do Pará e de Rondônia que alcançam valores superiores a $41.000 \mu \mathrm{g} . \mathrm{m}^{-3}$.

Ressalta-se que neste trabalho os dados de entrada no modelo extraídos dos focos de queimadas detectados pelos algoritmos presentes nos produtos WFABBA e MOD14/MYD14, correspondem a aproximadamente 506 mil focos detectados pelos produtos MOD14/MYD14 (Giglio et al., 2003) e 439 mil focos detectados pelo produto WFABBA (Prins et al., 1998). Ainda, para calcular a taxa de emissão de $\mathrm{PM}_{2,5 \mu \mathrm{m}}$, os dados obtidos a partir do coeficiente de consumo de biomassa foram multiplicados pelos fatores de emissão de cada espécie (Pereira et al., 2009).

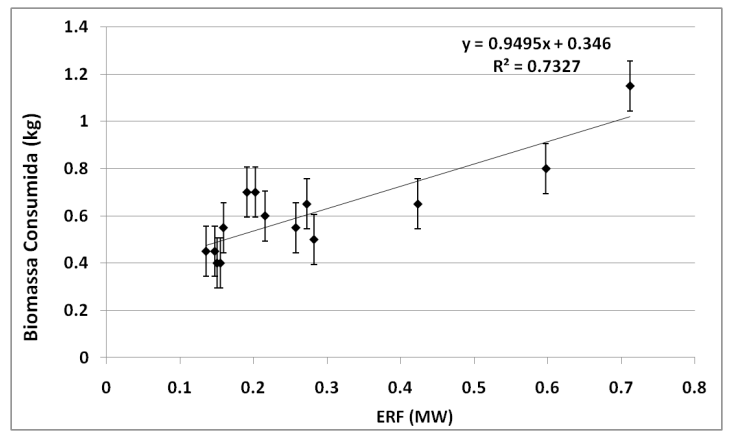

Figura 4 - Regressão linear e erro padrão para cada amostra relacionando o total de biomassa consumida e a ERF medida em laboratório. 

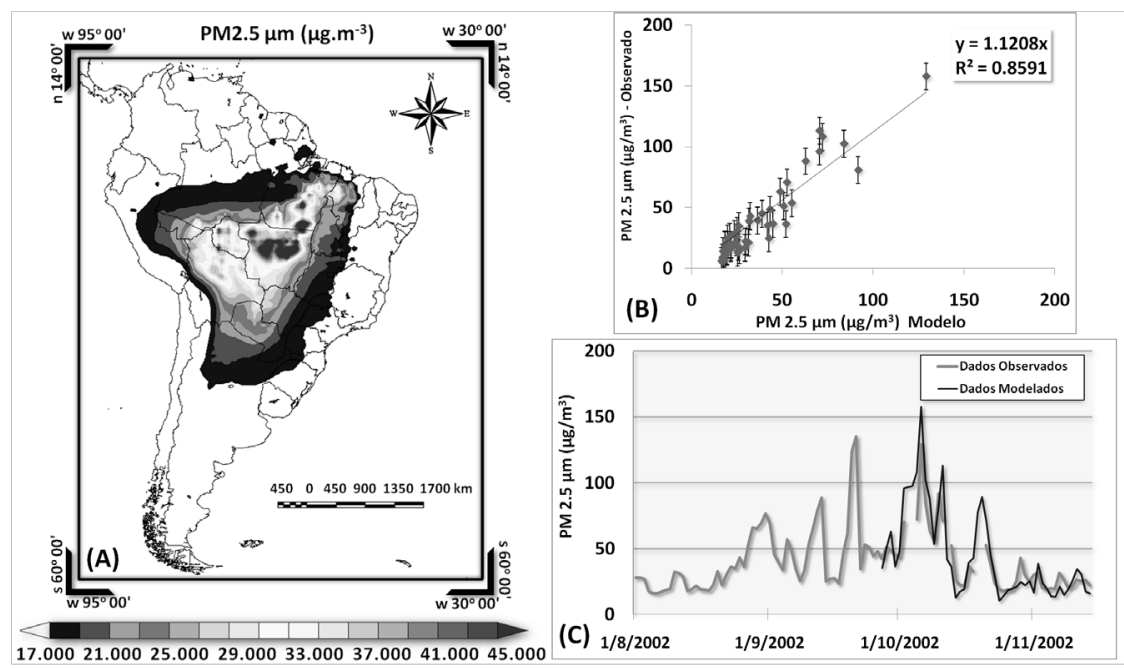

Figura 5 - (A) Mapa da emissão total de PM2,5 $\mu \mathrm{m}\left(\mu \mathrm{g} \cdot \mathrm{m}^{-3}\right)$ para o período de 15/Ju1/2002 a 15/Nov/2002; (B) Regressão linear entre o dado modelado e o dado observado com as barras de erro padrão; (C) Série temporal do PM2,5 $\mu \mathrm{m}\left(\mu \mathrm{g} \cdot \mathrm{m}^{-3}\right.$ ) observado (em preto) e o estimado pelo coeficiente obtido no experimento do LARAD/INPE (em cinza).

\subsection{Consumo de biomassa para a América do Sul}

A Figura 6 mostra o consumo diário de biomassa pelas queimadas, em teragramas (Tg), de 15/07/2002 a 26/11/2002 para a América do Sul. Os valores diários do consumo de biomassa correspondem aos valores integrados de todos os focos detectados pelo WFABBA/GOES a cada 30 minutos e as 4 passagens diárias do MODIS (10h30min e 22h30min - MODIS/Terra e 13h30min e 01h30min - MODIS/Aqua). Nota-se que mesmo na época de intensas queimadas, que ocorrem principalmente nos meses de agosto e setembro, na série temporal constatam-se algumas descontinuidades nos dados possivelmente devido à presença de nuvens. Desta forma, em alguns casos, a presença de nuvens e a falta de imageamento ocasionam baixos valores no consumo de biomassa.

Observa-se em cinza claro (Figura 6) a quantidade de biomassa consumida (conteúdo de carbono) estimada a partir dos dados de ERF do MODIS. Percebe-se que os dados diários de biomassa consumida estimados pelo MODIS são menores do que os dados estimados pelo GOES. Este fato está relacionado com a frequência de observações diárias. Enquanto que o MODIS obtém as informações de queimadas 4 vezes por dia, os dados provenientes do GOES são obtidos aproximadamente a cada 30 minutos. Destaca-se que os meses de agosto, setembro e outubro correspondem ao período de máxima incidência de focos de queimada, e consequentemente, de maior consumo de biomassa. Nestes, os valores podem superar $5 \mathrm{Tg}(5.000 .000 \mathrm{t})$, com uma média diária de $2,2 \mathrm{Tg}(0,8 \mathrm{Tg}$ estimados pelo MODIS e 1,32 Tg estimados pelo GOES).

A quantidade total de biomassa acima do solo na bacia amazônica apresenta variações significativas dependendo da metodologia adotada, em que fatores como a quantidade de carbono presente na vegetação e o seqüestro de carbono para o próprio crescimento da área queimada são difíceis de calcular. Saatchi et al. (2006) estimou a quantidade total de biomassa em aproximadamente $86 \mathrm{Pg}$ (petagramas $-10^{15}$ gramas) para a bacia amazônica, aproximadamente 300 a 400 toneladas por hectare (ha).

Diversos autores como, por exemplo, Tian et al. (1998), Fearnside e Barbosa (1998), Rosenfeld (1999), Houghton (1999), Houghton et al. (1999), Houghton et al. (2001), Houghton e Hackler (2006), Fernandes et al. (2007), Freitas et al. (2009) e Longo et al. (2007) analisaram a quantidade de biomassa consumida pelas queimadas na América do Sul e em outros continentes, porém um consenso sobre a quantidade de biomassa nestes ecossistemas está longe de se constatar. Entre os valores de biomassa consumida encontrados pelos autores acima mencionados, pode-se destacar o valor de $0,2 \mathrm{Pg}$ C.ano ${ }^{-1}$ encontrado por Houghton et al. (1999); e 0,3 Pg C.ano ${ }^{-1}$ encontrado por Tian et al. (1998).

O método originado a partir de análises da radiância emitida, da energia radiativa do fogo (ERF) e do consumo de biomassa originadas nos experimentos de combustão realizados no LARAD/INPE, obteve um total de 0,28 $\pm 0,001 \mathrm{Pg}$. Entre as principais vantagens deste método está a utilização de produtos em tempo quase-real para se estimar o total consumido pelas queimadas.

\section{CONSIDERAÇÕES FINAIS}

A quantização das emissões dos gases do efeito estufa provenientes da queima de biomassa é necessária para 


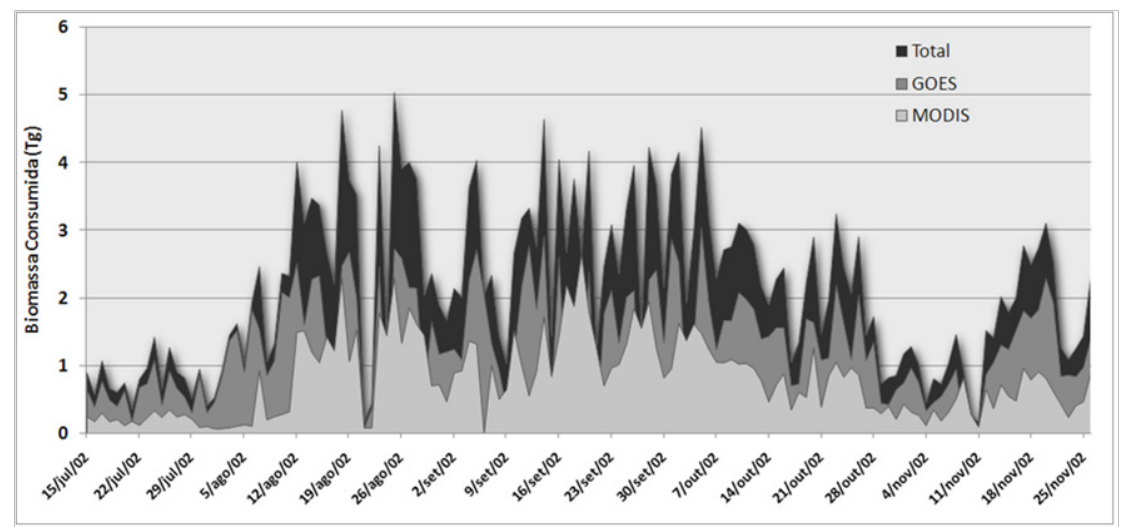

Figura 6 - Consumo diário de biomassa (Tg) ocasionado pelas queimadas na América do Sul (15/07/2002 a 26/11/2002) para os dados estimados pelo MODIS, pelo GOES e pela soma de ambos (total).

inventários anuais e sua estimativa a partir de dados derivados dos satélites ambientais é de fundamental importância para a modelagem do tempo e clima.

Com um coeficiente de determinação de 0,73 e correlação de $85 \%$ (significativos a 5\% pelo teste-t de Student), os experimentos de combustão controlada de biomassa vegetal de pequena escala realizados no INPE, permitem calcular o total de biomassa consumida pela queimada a partir da ERF. O coeficiente encontrado $(0,949)$ é aproximadamente $158 \%$ e $34 \%$ maior do que os encontrados anteriormente pelos métodos de Wooster et al. (2005) e Freeborn et al. (2008), respectivamente.

Embora as bibliografias sugiram que a quantidade de biomassa consumida é proporcional a ERF liberada, percebe-se que os três experimentos que foram realizados com espécies vegetais distintas, encontraram valores diferentes para cada ocasião. Desta forma, sugere-se que os experimentos de combustão de pequena escala sejam realizados com um número maior de espécies vegetais para verificar possíveis alterações em seus coeficientes.

\section{REFERÊNCIAS BIBLIOGRÁFICAS}

ANDREAE, M. O. Biomass Burning: its history, use, and distribution and its impact on environmental quality and global climate. In: Levine, J. S. (Ed.). Global Biomass Burning: Atmospheric, Climatic, and Biospheric Implications, The MIT Press, Cambridge, MA, p. 2-21, 1991.

ANDREAE, M. O.; MERLET, P. Emission of trace gases and aerosols from biomass burning, Global Biogeochem. Cycles, n. 15, v.4, p. 955-966, 10.1029/2000GB001382, 2001.

ANDREAE, M.; ROSENFELD, D.; ARTAXO, P.; COSTA, A.; FRANK, G.; LONGO, K. M.; SILVA DIAS, M. A. F. Smoking rain clouds over the Amazon, Science, v. 303, p. 1342-1345, 2004.
ARAÚJO, T. M.; CARVALHO Jr., J. A.; HIGUCHI, N.; BRASIL Jr., A. C. P.; MESQUITA, A. L. A. A tropical rainforest clearing experiment by biomass burning in the state of Pará, Brazil. Atmospheric Environment, n. 33, p. 1991 - 1998, 1999.

BADARINATH, K. V. S.; LATHA, K. M.; CHAND, T. R. K.; GUPTA, P. K.; GHOSK, A. B.; JAIN, S. L.; GERA, B. S.; SINGH, R.; SARKAR, A. K.; SINGH, N.; PARMAR, R. S.; KOUL, S.; KOHLI, R.; NATH, S.; OJHA, V. K.; SIGH, G. Characterization of aerosols from biomass burning - a case study from Mizoram (Northeast), India. Chemosphere, n. 54, p. 167-175, 2004.

CHU, D. A.; KAUFMAN, Y. J.; REMER, L. A.; HOLBEN, B. N. Remote sensing of smoke from MODIS airborne simulator during the SCAR-B experiment, Journal of Geophysical Research, v. 103, n. 24, p. 31979-31987, 1998.

DOZIER, J. A method for satellite identification of surface temperature fields of subpixel resolution. Remote Sensing of Environment, v. 11, p. 221-229, 1981.

FEARNSIDE, P. M.; BARBOSA, R. I. Soil carbon changes from conversion of forest to pasture in Brazilian Amazonia. For. Ecol. Mgmt, v. 108, p. 147-166, 1998.

FERNANDES, S. D.; TRAUTMANN, N. M.; STREETS, D. G.; RODEN, C. A.; BOND, T. C. Global biofuel use, 1850/2000. Global Biogeochemical Cycles, v. 21, n.2, GB2019, 2007.

FREEBORN, P. H.; WOOSTER, M. J.; HAO, W. M.; RYAN, C. A.; NORDGREN, B. L.; BAKER, S. P.; ICHOKU, C. Relationships between energy release, fuel mass loss, and trace gas and aerosol emissions during laboratory biomass fires, J. Geophys. Res., v. 113, n. D1, D01102, 10.1029/2007JD008489, 2008.

FREITAS, S. R.; LONGO, K. M.; SILVA DIAS, M. A. F.; CHATFIELD, R.; SILVA DIAS, P.; ARTAXO, P.; ANDREAE, M. O.; GRELL, G.; RODRIGUES, L. F.; FAZENDA, A.; PANETTA, J. The Coupled Aerosol and 
Tracer Transport model to the Brazilian developments on the Regional Atmospheric Modeling System (CATT-BRAMS) - Part 1: Model description and evaluation, Atmos. Chem. Phys., v. 9, p. 2843-2861, 2009.

GALANTER, M.; LEVY II, H.; CARMICHAEL, G. R. Impacts of biomass burning on tropospheric $\mathrm{CO}, \mathrm{NO}_{\mathrm{x}}$ and $\mathrm{O}_{3}$. Journal of Geophysical Research, v. 105, n. D5, p. 6633-6653, 2000.

GIGLIO, L.; KENDALL, J.; MACK, R. A multi-year active fire dataset for the tropics derived from the TRMM VIRS, International Journal of Remote Sensing, v. 24, p. 45054525, 2003.

GIGLIO, L. MODIS Collection 4 Active Fire Product User's Guide. Version 2.2, 2005. Disponível em <maps.geog.umd. edu/products/MODIS_Fire_Users_Guide_2.2.pdf $>$. Acesso em 26/01/2007.

HAO, W. M.; LIU, M. H. Spatial and temporal distribution of tropical biomass burning. Global Biogeochem. Cycles, vol. 8, pp. 495-503, 1994.

HOUGHTON, R. A. The annual net flux of carbon to the atmosphere from changes in land use 1850-1990, Tellus, v. 51, n.2, p. 298-313, doi:10.1034/j.1600-0889.1999.00013.x, 1999.

HOUGHTON, R. A.; SKOLE, D. L.; NOBRE, C. A.; HACKLER, J. L.; LAWRENCE, K. T.; CHOMENTOWSKI, W. H. Annual fluxes of carbon from deforestation and regrowth in the Brazilian Amazon, Nature, v. 403, p. 301304, doi:10.1038/35002062, 1999.

HOUGHTON, R. A.; LAWRENCE, K. T.; HACKLER, J. L.; BROWN, S. The spatial distribution of 10 forest biomass in the Brazilian Amazon: a comparison of estimates, Global Change Biol., v. 7, p. 731-746, 2001.

HOUGHTON, R. A.; HACKLER, J. L. Emissions of carbon from land use change in sub-Saharan Africa, Journal of Geophysical Research, v. 111, 2006.

ICHOKU, C.; KAUFMAN, Y. J. A method to derive smoke emission rates from MODIS fire radiative energy measurements. IEEE Trans. on Geosc. \& Rem. Sens., v. 43, n. 11, p. 2636-2649, 2005.

INTERGOVERNAMENTAL PANEL ON CLIMATE CHANGE (IPCC). Contribution of Working Group I to the Fourth Assessment Report of the Intergovernmental Panel on Climate Change, New York : Cambridge Univ. Press, 2007.

KAUFMAN, Y. J.; SETZER, A. W.; WARD, D.; TANRÉ, D.; HOLBEN, B. N.; MENZEL, P.; PEREIRA, M. C.; RASMUSSEN, R. Biomass Burning and Spaceborne Experiment in the Amazonas (BASE-A). Journal of Geophysical Research, v. 97, n. D13, p. 14581-14599, 1992.

KAUFMAN, Y. J.; REMER, L.; OTTMAR, R.; WARD, D.; RONG-R, L.; KLEIDMAN, R.; FRASER, R.; FLYNN, L.;
MCDOUGAL, D.; SHELTON, G. Relationship between remotely sensed fire intensity and rate of emission of smoke: SCAR-C experiment. In: Levine, J. (Ed.), Global biomass burning, MA: MIT Press, p. 685-696, 1996.

KAUFMAN, Y. J.; JUSTICE, C. O.; FLYNN, L. P.; KENDALL, E. M. P.; GIGLIO, L.; WARD, D. E.; MENZEL, W. P.; SETZER, A. W. Potencial global fire monitoring from EOSMODIS, Journal of Geophysical Research, v. 103, n. 24, p. 32215-32238, 1998a.

KAUFMAN, Y. J.; KLEIDMAN, R. G.; KING, M. D. SCAR-B fires in the tropics: Properties and remote sensing from EOSMODIS, Journal of Geophysical Research, v. 103, n. 24, p. 31955-31968, 1998b.

LEVINE, J. S. Biomass burning and the production of greenhouse gases. In: Zepp, R. G. (Ed.) Climate Biosphere Interaction: Biogenic Emissions and Environmental Effects of Climate Change, Isbn 0-471-58943-3, 1994. Disponível em: http://asd-www.larc.nasa.gov/biomass_burn/biomass. html. Acesso em 24/01/2007.

LONGO, K.; FREITAS, S. R.; SETZER, A.; PRINS, E.; AARTAXO, P.; ANDREAE, M. The Coupled Aerosol and Tracer Transport model to the Brazilian developments on the Regional Atmospheric Modeling System (CATT-BRAMS). Part 2: Model sensitivity to the biomass burning inventories, Atmos. Chem. Phys. Discuss., p. 8571-8595, 2007.

MORAES, E. C.; FRANCHITO, S. H.; BRAHMANANDA RAO, V. Effects of biomass burning in Amazonia on climate: A numerical experiment with a statistical-dynamical model, Journal of Geophysical Research, v. 109, n. D05109, p. 1-12, 2004.

PEREIRA, G.; FREITAS, S. R.; MORAES, E. C. Estimating trace gas and aerosol emissions over South America: Relationship between fire radiative energy released and aerosol optical depth observations. Atmospheric Environment, v. 43, n. 40, p. 6388-6397. doi: 10.1016/j. atmosenv.2009.09.013, 2009.

PRINS, E. M.; FELZ, J. M.; MENZEL, W. P.; WARD, D. E. An overview of GOES- 8 diurnal fire and smoke results for SCAR-B and 1995 fire season in South America, Journal of Geophysical Research, v. 103, n. D24. p. 31821-31825, 1998.

ROSENFELD, D. TRMM observed first direct evidence of smoke from forest fires inhibiting rainfall, Geophys. Res. Lett., v. 26, p. 3105- 3108, 1999.

SAATCHI, S. S.; HOUGHTON, R. A.; ALVALÁ, R. C. S.; SOARES, J. V.; YU, Y. Distribution of Aboveground Live Biomass in the Amazon Basin, 2006. Disponível em: $<$ http:// www-radar.jpl.nasa.gov/carbon/ab/fbc.htm>. Acesso em: 23/03/2008

SETZER, A. W.; PEREIRA, M. C. P; ALMEIDA, S. O. Relatório de atividades do projeto IBDF-INPE "SEQE"- 
ano 1987. . -. SAO JOSE DOS CAMPOS, SP: INPE, 1988. 101 p., $93204 \mathrm{~cm}$. (INPE-4534-RPE/565).

SETZER, A. W.; PEREIRA, M. C. P. Relatório de atividades do projeto "SEQE" - Ano 1988. São José dos Campos: Instituto Nacional de Pesquisas Espaciais, 1988. (INPE).

SILVA, A. S. da; SILVA, M. C. da. Prática de queimadas e as implicações sociais e ambientais na cidade de Araguaina-TO. Revista Caminhos de Geografia, v. 18, pg. 8 - 16, 2006.

TIAN, H.; MELillo, J. M.; KICKLIGHTER, D. W.; MCGUIRE, A. D.; HELFRICH, J. V. K.; MOORE, B.; VÖRÖSMARTY, C. J.. Effect of interannual climate variability on carbon storage in Amazonian ecosystems. Nature, v. 396, p. 664- 667, 1998.

WALKO, R.; BAND, L.; BARON, J.; KITTEL, F.; LAMMERS, R.; LEE, T.; OJIMA, D.; PIELKE, R.; TAYLOR, C.; TAGUE, C.; TREMBACK, C.; VIDALE, P.. Coupled atmosphere-biophysics-hydrology models for environmental modeling, J. Appl. Meteorol., v. 39, p. 931-944, 2000.

WERF, G. R.; RANDERSON, J. T.; GIGLIO, L.; COLLATZ, G. J.; KASIBHATLA, P. S.; ARELLANO JR., A. F. Interannual variability in global biomass burning emissions from 1997 to 2004, Atmospheric Chemistry and Physics, n. 6 , p. 3423-3441, 2006.
WOOSTER, M. J.; ROBERTS, G.; PERRY, G.; KAUFMAN, Y. J. Retrieval of biomass combustion rates and totals from fire radiative power observations: calibration relationships between biomass consumption and fire radiative energy release, Journal of Geophysical Research, v. 110, n. D21111: doi: 10.1029/2005JD006318, 2005.

WOOSTER, M. J.; ROTHERY, D. A. Thermal monitoring of Lascar Volcano, Chile using infrared data from the Along Track Scanning Radiometer: A 1992 - 1995 time series, Bulletin of Volcanology, n. 58, p. 566-579, 1997.

WOOSTER, M. J.; ZHUKOV, B.; OERTEL, D. Fire radiative energy for quantitative study of biomass burning: derivation from the BIRD experimental satellite and comparison to MODIS fire products. Remote Sensing of Environment, n. 86, p. $83-107,2003$. 